\title{
Efficient Method for Detecting and Localizing Concept Drifts from Event Logs In Process Mining
}

\author{
Trupti Kakkad $^{1}$, Dr. Rahila Sheikh ${ }^{2}$
}

\author{
${ }^{1,2}$ Rajiv Gandhi College of Engineering and Research Technology Chandrapur, Babupeth, Chandrapur -442403
}

\begin{abstract}
Although most business processes modification over time, modern process mining techniques tend to survey these processes as if they are in a balanced state. The change in processes may be immediately or moderately. The drift could also be periodic (e.g., due to seasonal influences) or one-of-a-kind (e.g., the consequences of recent legislation). To find and perceive such idea drifts in processes is crucial for the process management. A generic framework and specific techniques to notice once a method changes and to localize the components of the method that have modified are given during this paper. To delineate relationships among activities, completely different options are projected. Variations between consecutive populations are discovered by victimization these options. Like as a plugin of the promenade methodology mining framework, The approach has been implemented and has been evaluated using every simulated event data exhibiting controlled conception drifts and from a Dutch municipality, real-life event information.
\end{abstract}

Keywords: Concept drift, flexibility, hypothesis tests, process changes, process mining;

\section{Introduction}

Business processes are nothing over logically connected tasks that use the resources of a corporation to achieve an outlined business results. Work type for business can also be viewed from a variety of views, including the management flow, data, and also the resource views. In today's dynamic marketplace, to reduce cost and to improve performance is necessary for enterprises to streamline their processes and also customers expect organizations to be flexible and adapt to changing circumstances.

Like the WABO act new legislations and conjointly the Sarbanes-Oxley Act, in offer and demand extreme Variations, seasonal effects, natural calamities and disasters, purpose in time escalations, and so on, to change their processes are forcing organizations. As an example, throughout a disaster, hospitals, and for his or her operative procedures banks modification. The power to react and adapt to changes in its operative surroundings describes economic success of a corporation.

An abundance of event logs several of today's data systems are recording. Method mining may be a comparatively young analysis discipline geared toward discovering, monitoring, and rising real processes by extracting data from event logs. For instance, once discovering a method model from event logs, it's assumed that \{the method the method\} at the start of the recorded amount is that the same because the process at the tip of the recorded amount. As mentioned earlier, for to adapt to dynamical circumstances processes would possibly modification. Conception drift refers to things during which the method is ever-changing while being analyzed. There's a requirement for techniques that cope with such second-order dynamics. Analyzing such changes is of utmost importance once supporting or rising operational methods and to get a correct insight on process executions at any instant of your time.

When coping with conception drifts in method mining, the subsequent three main challenges emerge: Change purpose detection:

The first and most basic drawback is to discover conception drift in processes, i.e., to notice that a method modification has taken place. As an example, by analyzing an incident log from a corporation (deploying seasonal processes), we should always be able to discover that method changes happen which the changes happen at the onset of a season.

Change Localization and Characterization:

Once some extent of modification has been known, following step is to characterize the character of change, and determine the region(s) of modification (localization) during a method. For example, within the example of a seasonal method, the modification might be that a lot of resources are deployed or that special offers are provided throughout vacation seasons.

\section{Change Method Discovery}

Having known, localized, and characterized the changes, it's necessary to place all of those in perspective. For techniques/tools that exploit there's a demand and relate these discoveries. Unravelling the evolution of a method should end in the invention of the modification method describing the second-order dynamics. We can differentiate between two broad classes of dealing with concept drifts when analyzing event logs:

\section{1) Offline analysis:}

This refers to the scenario where the presence of changes or the occurrence of drifts need not be uncovered in a real time. This is acceptable in cases wherever the detection of changes is usually utilized in post-mortem analysis, the results of which might be thought of when designing/improving processes for later readying. For instance, offline idea drift analysis may be used to better cope with seasonal effects (hiring less employees in summer or skipping checks within the weeks before Christmas).

\section{2) Online analysis:}

This refers to the situation wherever changes have to be

\section{Volume 5 Issue 1, January 2016}




\section{International Journal of Science and Research (IJSR) \\ ISSN (Online): 2319-7064 \\ Index Copernicus Value (2013): 6.14 | Impact Factor (2014): 5.611}

compelled to be discovered in close to real time. This can be applicable in cases wherever a company would be a lot of interested in knowing a modification within the behavior of their customers or a modification in demand as and once it's happening. Such time period triggers (alarms) can modify organizations to require fast remedial actions and avoid any repercussions.

\section{Literature Survey}

[1] W. M. P. van der Aalst, M. Rosemann, and M. Dumas, "Deadline based increase in process aware data systems," decision Support Syst., vol. 43, no. 2, pp. 492-511, 2011.Decision making in process-aware info systems involves build-time and run-time choices. At build-time, supported the organization's objectives good methodology models are designed, infrastructure, context, constraints, etc. At run-time, this perfect view is usually broken. Specifically, method models typically assume that planned activities happen at intervals a particular amount. Once such assumptions aren't fulfilled, users should build selections relating to different arrangements to realize the goal of finishing the method within its expected timeframe or to reduce timing. We tend to seek advice from the specified selections as escalations. From the progress management field for escalations this paper proposes a framework that pulls on established principles. The paper identifies and classifies variety of increase mechanisms such as: changing the routing of labor, dynamical the work distribution, or changing the necessities with relation to accessible knowledge. To illustrate and live these mechanisms a case study and a simulation experiment arqwon't

[2] B. F. van Dongen and W. M. P. van der Aalst, "A meta model for process mining knowledge" in Proc. vol. 2.

2005, pp. 309-320.Modern process-aware info systems store careful information regarding processes as they're being dead. This type of knowledge may be used for terribly totally different functions to extract information (e.g., inside the variability of models) from this the term method mining refers to the techniques and tools. many key players during this space have developed refined method mining tools, like Aris PPM and therefore the power unit Business Cockpit, that are capable of using the data obtainable to come up with significant insights. Although, the approach presented during this paper is extremely pragmatic, it may be seen as a primary step towards and ontological analysis of method mining information.

[3] F. Daniel, S. Dustdar, and K. Barkaoui, "Process mining manifesto," inBPM 2011 Workshops, vol. 99. New

York, NY, USA: Springer-Verlag, 2011, pp. 169-194. By a bunch of people a manifesto is also a "public declaration of principles and intentions". On methodology mining this manifesto is written by members and supporters of the IEEE Task Force. To plug the analysis is that the goal of this task force, development, education, implementation, evolution, and understanding of methodology mining. To push the topic of method mining The IEEE Task Force on method Mining aims. The method mining manifesto inside the context of this task force, a bunch of over seventy five people involving over fifty organizations created. By process a collection of guiding principles and listing necessary challenges, for code developers this declaration hopes to operate a guide, scientists, consultants, business managers, and end-users. To reinforce the (re)design, control, and support of operational business processes, the goal is to increase the maturity of method mining as a novel tool.

[4] R. P. J. C. Bose, W. M. P. van der Aalst, I. Žliobait'e, and M. Pechenizkiy, "Handling idea drift in method mining," in Proc. Int. CAiSE, 2011, pp. 391-405. To adapt to impulsive circumstances Operational processes need to modification, e.g., new legislation, extreme variations in offer and demand, seasonal effects, etc. Whereas the subject of flexibility is well-researched within the gait domain, modern method mining approaches assume the method to be in steady state. Once discovering a method model from event logs, it's assumed that the method at the start of the recorded amount is that the same as the process at the top of the recorded amount. Due to the event observed as conception drift clearly, this can be often not the case whereas cases arq being handled, the method itself could also be dynamical. This paper presents associate degree approach to analyses such second-order dynamics. In ProM3 and evaluated by analyzing an evolving methodology the approach has been enforced.

[5] I. Žliobait"e, "Learning below conception drift: a summary "CoRR, vol. abs/1010.4784, 2010.Thought drift refers to a non-stationary learning drawback over time. The coaching and the application information typically mismatch in real world issues. During this report we tend to present a context of thought drift problem 1 . We tend to concentrate on the problems relevant to adaptive coaching set formation. We tend to present the framework and language, and formulate a world image of thought drift learners design. We tend to begin with formalizing the framework for the thought drifting knowledge in Section one. In Section two we tend to discuss the adaptively mechanisms of the thought drift learners. In Section three we tend to summary the principle mechanisms of thought drift learners. During this chapter we tend to provides a general image of the out there algorithms and reason them supported their properties. Section five discusses the connected analysis fields and Section five teams and presents major thought drift applications. This report is meant to relinquish a bird's view of thought drift analysis field, give a context of the analysis and position it at intervals broad spectrum of analysis fields and applications.

[6] C. Alippi and M. Roveri, "Just-in-time adaptive classifiers-Part I: Detecting nonstationary changes," IEEE Trans. Neural Netw. vol. 19, no. 7, pp. 1145-1153, Jul. 2008. The stationarity requirement for the process generating the data is a common assumption in classifiers' design. When such hypothesis does not hold, e.g., in applications affected by aging effects, drifts, deviations, and faults, classifiers must react just in time, i.e., exactly when needed, to track the process evolution. The first step in designing effective just-in-time classifiers requires detection of the temporal instant associated with the process change, and the second one needs an update of the knowledge base used by the classification system to track the process evolution. This paper addresses the change detection aspect leaving the design of just-in-time adaptive classification systems to a 


\section{International Journal of Science and Research (IJSR) \\ ISSN (Online): 2319-7064 \\ Index Copernicus Value (2013): 6.14 | Impact Factor (2014): 5.611}

companion paper. Two completely automatic tests for detecting nonstationary phenomena are suggested, which neither require a priori information nor assumptions about the process generating the data. In particular, an effective computational intelligence-inspired test is provided to deal with multidimensional situations, a scenario where traditional change detection methods are generally not applicable or scarcely effective.

[7]C. Alippi, G. Boracchi, and M. Roveri, "Just-in-time classifiers for recurrent ideas" IEEE Trans. Neural Netw. Learn. Syst., vol. 24, no. 4, pp. 620-634, Apr. 2013.To conception drift in evolving environments Just-in-time (JIT) classifiers operate by classifying instances and reacting. In stationary conditions, by exploiting additional supervised Knowledge returning from the arena a JIT classifier improves its accuracy over time. In nonstationary conditions, however, the classifier reacts as shortly as conception drift is detected; this classification setup is discarded and to remain the accuracy high an appropriate one activated. We present a completely unique generation of JIT classifiers ready to cope with repeated conception drift by means of a sensible formalization of the conception illustration and the definition of a collection of operators functioning on such representations. When needed by considering change-detection tests observance every inputs and classes distributions to changes specifically the conceptdrift detection activity, that's crucial in promptly reacting, is advanced.

[8] R. Elwell and R. Polikar, "Incremental learning of conception drift in nonstationary environments," IEEE Trans.

Neural Netw. vol. 22, no. 10, pp. 1517-1531, Oct. 2011. For progressive learning of conception drift we tend to introduce an ensemble of classifiers-based approach, defined by nonstationary environments (NSEs), where over time the underlying data distributions modification. The planned algorithmic rule, named Learn++.NSE, learns from consecutive batches of data on the character or rate of drift whereas not making any assumptions; it'll learn from such environments that have constant or variable rate of drift, addition or deletion of conception classes, conjointly as alternate drift. The algorithmic rule learns incrementally, for the Learn++ family of algorithms as totally different members, that is, to decadently seen data whereas not requiring access. For each batch of data it receives Learn++.NSE trains one new classifier, and combines these classifiers using a dynamically weighted majority vote. In determinant the vote weights is that the novelty of the approach, on current and past environments supported each classifier's time-adjusted accuracy. This approach permits the algorithmic rule to acknowledge, and act consequently, to the changes in underlying information distributions, also on a doable reoccurrence of an earlier distribution. We tend to evaluate the algorithmic rule on many artificial datasets designed to simulate a spread of non-stationary environments, also as a real-world weather prediction dataset. Comparisons with many different approaches are enclosed. Results indicate that for the dynamic environments very closely Learn++.NSE can track, and for conception drift despite the type. To permit future use, comparison and benchmarking by interested researchers, we tend to conjointly unleash our information employed in this paper.

[9] A. Tsymbal, M. Pechenizkiy, P. Cunningham, and S. Puuronen, "Handling local conception drift with dynamic integration of classifiers: Domain of antibiotic resistance in medical building infections," in Proc. nineteenth IEEE Int. Symp. CBMS, Nov. 2006, pp. 679-684. The drawback, called conception drift is, within the planet ideas and knowledge distributions are typically not stable however modification with time, from knowledge and needs special approaches complicates the task of learning a model, totally different from unremarkably used techniques, to the target conception that treat inward instances as equally necessary contributors. Ensemble learning is among the foremost wellliked and effective approaches to handle conception drift, wherever a collection of models engineered over totally different time periods is maintained and therefore the best model is chosen or the predictions of models are combined. During this paper at the instance level we tend to contemplate the employment of an ensemble integration technique that helps to raised handle conception drift. for handling conception drift with ensembles Our experiments with real-world antibiotic resistance knowledge demonstrate that dynamic integration of classifiers engineered over little time intervals is simpler than globally weighted option that is presently the foremost unremarkably used integration approach.

[10] A. J. M. M. Weijters and W. M. P. van der Aalst, "Rediscovering workflow models from eventbasedknowledge victimization very little thumb," Integer. Compute. Aided Eng., vol. 10, no. 2, pp. 151-162, 2003.Contemporary work flow management systems are driven by specific method models, i.e., a totally given progress design is needed so as to enact a given progress process. Making a workflow design may be a sophisticated time-consuming method and usually, there are discrepancies between the particular work flow processes and also the processes as perceived by the management. Therefore, we have a tendency to propose a way for rediscovering work flow models. This system uses work flow logs to get the work flow method because it is truly being dead. The work flow log contains data concerning events happening. We assume that these events are completely ordered and every event refers to one task being executed for one case. This data will simply be extracted from transactional info systems (e.g., Enterprise Resource planning systems like SAP and Baan). The rediscovering technique projected during this paper will cope with noise and might even be used to validate work flow processes by revealing and measuring the discrepancies between prescriptive models and actual process executions.

\section{Framework and Design}

We have projected the framework for analyzing concept drifts in process mining as shown in below Fig. 


\section{International Journal of Science and Research (IJSR)}

ISSN (Online): 2319-7064

Index Copernicus Value (2013): 6.14 | Impact Factor (2014): 5.611

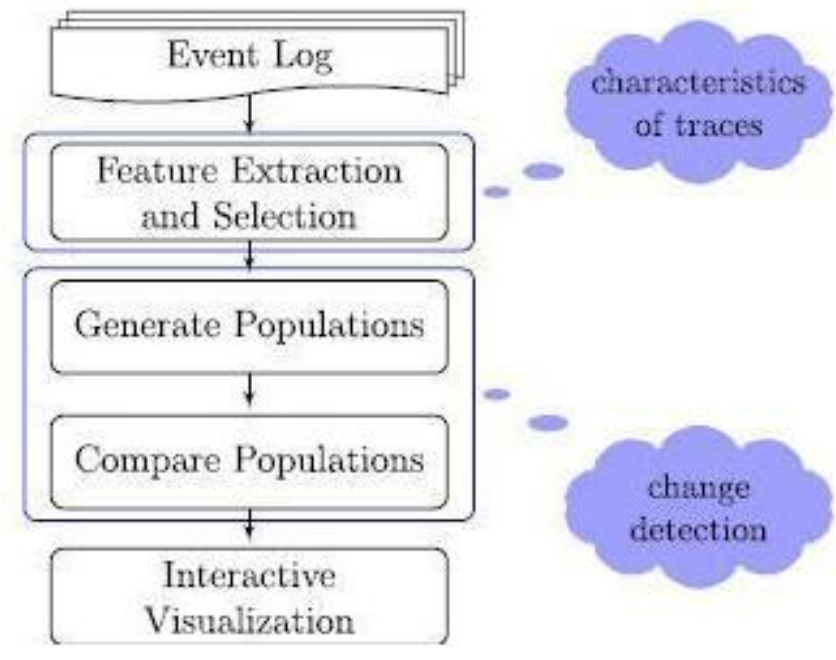

The framework identifies the following steps:

1) Feature extraction and selection: in process the characteristics of the traces in an incident log this step pertains. During this paper, for method instances we've got outlined four options that characterize the control-flow perspective. In an incident log. betting on the main target of research, we tend to could outline extra options, e.g., in analyzing changes if we tend to have an interest in organizational/resource perspective, from social networks as a way for characterizing the event log we tend to could contemplate options derived. Additionally to feature extraction, this step additionally involves feature choice. Feature choice is very important once the quantity of options extracted is giant. To handle high dimensionality we tend to could think about dimensionality reduction techniques like PCA or random projection.

2) Generate populations: within the previous step supported the options selected an incident log may be reworked into an information stream. For learning the changes within the characteristics of traces this step deals with process the sample populations. For generating these populations from the info stream completely different criteria/scenarios

Could also be thought-about. for handling gradual and revenant drifts we tend to may additionally consider, as an example, non-continuous windows (there may be a gap between 2 populations), adaptive windows (windows may be of various lengths), and so on, that are additional applicable.

3) Compare populations: for any modification in characteristics once the sample populations are generated, succeeding step is to investigate these populations. During this paper, for comparison populations we tend to advocate the utilization of statistical hypothesis tests. The null hypothesis in statistical tests states that distributions (or suggests that, or normal deviations) of the 2 sample populations are equal. Completely different statistical tests may be used betting on desired assumptions and therefore the focus of research,

4) Interactive visualization: to an analyst the results of comparative studies on the populations of trace characteristics may be intuitively presented. As an example, as a drift plot the importance possibilities of the hypothesis tests may be pictured. Within the characteristics of traces Troughs in such a drift plot signify a modification within the significance chance thereby implying a modification.

5) Analyze modifications: image techniques like the drift plot will assist in characteristic the change points. In characterizing and localizing the modification and in discovering the amendment method having known that a modification had taken place, this step deals with techniques that assist an analyst.

\section{Implementation Details}

The Ideas Projected in this paper have been realized as the concept drift plug-in in the ProM6 framework. For method mining visualized to provide to analysis and image of results a typical basis Promise a plug-able surroundings for every kind of method mining techniques ranging from importing, exporting, and filtering event logs (process models).Over years, for method mining promenade has emerged to be the actual normal. Within the projected framework the conception drift plug-in implements all of the steps and may be merely extended with extra parts (e.g., new options may be easily added). For the hypothesis tests as a drift plot the plug-in supports visualization of the significance probability.

\section{Conclusion}

Based on event logs the thought drift in method mining i.e. explore method changes. To find the modification in event logs the feature set and techniques are planned and during a method establish the regions of modification. Initially, our results show that with by sleuthing thought drifts no uniformity of cases arising thanks to method changes will be effectively dealt.

After identifying the change points, partition and auditing can be done. With modifications in any strategist perceptive this can be the start towards the dealing and analysis efforts. For to thought of as sudden and gradual drifts, the changes only with relation to the control-flow perspective manifested are. Hence, this is starting point for a new subfield in the process mining domain and there are other challenges that to be addressed such as change-pattern specific features, feature selection, Holistic approaches, recurring drifts, change process discovery ,sample complexity, online drift detection.

\section{References}

[1] W. M. P. van der Aalst, M. Rosemann, and M. Dumas, "Deadline-based escalation in process-aware information systems," DecisionSupportSyst., vol. 43, no. 2, pp. 492-511, 2011.

[2] B. F. van Dongen and W. M. P. van der Aalst, "A meta model for process mining data," in Proc. CAiSE Workshops (EMOI-INTEROPWorkshop),

[3] F. Daniel, S. Dustdar, and K. Barkaoui, "Process mining manifesto," inBPM 2011 Workshops, vol. 99. New York, NY, USA: Springer-Verlag, 2011, pp. 169-194.

[4] R. P. J. C. Bose, W. M. P. van der Aalst, I. Žliobait"e, and M. Pechenizkiy, "Handling concept drift in process mining," in Proc. Int. CAiSE, 2011,pp. 391-405. 


\section{International Journal of Science and Research (IJSR) \\ ISSN (Online): 2319-7064}

Index Copernicus Value (2013): 6.14 | Impact Factor (2014): 5.611

[5] I. Žliobait"e, "Learning under concept drift: An Overview, "CoRR, vol. abs/1010.4784, 2010.

[6] C. Alippi and M. Roveri, "Just-in-time adaptive classifiers-Part I: Detecting nonstationary changes," IEEE Trans. Neural Netw., vol. 19, no. 7, pp. 11451153, Jul. 2008.

[7] C. Alippi, G. Boracchi, and M. Roveri, "Just-in-time classifiers for recurrent concepts," IEEE Trans. Neural Netw. Learn. Syst., vol. 24, no. 4, pp. 620-634, Apr. 2013.

[8] R. Elwell and R. Polikar, "Incremental learning of concept drift in nonstationary environments," IEEE Trans. Neural Netw., vol. 22, no. 10, pp. 1517-1531, Oct. 2011.

[9] A. Tsymbal, M. Pechenizkiy, P. Cunningham, and S. Puuronen, "Handling local concept drift with dynamic integration of classifiers:Domain of antibiotic resistance in nosocomial infections," in Proc. 19th IEEE Int. Symp. CBMS, Nov. 2006, pp. 679-684.

[10]A. J. M. M. Weijters and W. M. P. van der Aalst, "Rediscovering workflow models from event-based data using little thumb,"

[11] Integer. Compute. Aided Eng., vol. 10, no. 2, pp. 151162, 2003. 